Check for updates

Cite this: RSC Adv., 2019, 9, 10733

Received 14th January 2019

Accepted 29th March 2019

DOI: $10.1039 / \mathrm{c} 9 \mathrm{ra00322c}$

rsc.li/rsc-advances

\title{
Soft ionic liquid multi-point touch sensor
}

\author{
Jarred Fastier-Wooller, (D) *a Toan Dinh, (D) ab Van Thanh Dau (D) a \\ and Dzung Viet Dao (D) ab
}

The development of electronic skin (e-skin) and soft tactile sensing has recently attracted great interest. Here we report for the first time on a novel ionic liquid (IL) based soft pressure sensor with multi-point touch detection capability using 1-butyl-3-methylimidazolium tetrafluoroborate $\left([\mathrm{BMIM}]^{+}\left[\mathrm{BF}_{4}\right]^{-}\right)$as a highly conductive sensing medium. The sensing mechanism is attributed to the repopulation of charge cations and anions in aqueous solution under pressure. The sensor can detect two dimensional touching positions with a sensitivity of $-0.28 \% \mathrm{kPa}^{-1}$. Our sensor showed good stability and temperature independence thanks to the incompressibility of $\mathrm{IL}$ in the range of touch pressure and the appropriate signal measurement configuration. We successfully demonstrated the sensor's capability to detect multipoint human touch with different pressure levels. Our simple design with smart structures and ease of fabrication processes enable the development of a soft and low-cost sensor with multiple-point sensing capabilities on a single chip.

\section{Introduction}

Soft sensors are used in various applications in robotics ${ }^{1-5}$ and as wearables. ${ }^{6-9}$ These sensors have the necessary properties and even show benefits over other sensors in their flexibility, stretchability, and sensitivity. ${ }^{10-13}$ Soft sensors possess the ability to measure pressure on a surface that is soft and flexible enough to mimic the dexterity and delicacy of human touch. ${ }^{\mathbf{1 4}, 15}$ Such sensors allow for a more advanced and versatile robot to object interaction and even human to robot interaction. ${ }^{16}$

Human touch is not solely dependent on pressure sensitivity; there is also thermal, pain, and visual feedback. These senses are crucial to the interaction and manipulation of objects. Though, before giving robots a sense of pain, it's important to make sure there is enough information in terms of touch. As processing power increases and more and more processing dependant solutions are presented, such as image processing, ${ }^{17,18}$ there is still a need for sensitive e-skins and soft tactile sensors that are of simple design and remain low-cost for easy fabrication, yet still allowing integration into more complex systems.

It has been discussed and shown that in order to perform dexterous manipulation of an object it is important to be able to detect force/pressure and position to better control slippage or sliding, ${ }^{15,19,20}$ or pressure distribution which has been given much attention in e-skin technologies. ${ }^{21-25}$ These solutions use an area or matrix of interconnected or individually connected

${ }^{a}$ School of Engineering and Built Environment, Griffith University, Southport, QLD 4222, Australia. E-mail: jarred.fastier-wooller@griffithuni.edu.au

${ }^{b} Q L D$ Micro- and Nanotechnology Centre, Griffith University, Brisbane, QLD 4111, Australia sensors to detect and map pressure areas. Many of these sensing devices provide separate sensors, each capable of performing a task such as temperature and pressure stacked matrices. ${ }^{26}$

This work presents a low-cost and simple fabrication process for a soft and localised pressure distribution sensor. Fabrication is performed using well established and simple fabrication methods whilst maintaining a simple yet effective structure. Thus, we avoid as many IC and MEMS processes or materials such as $\mathrm{CNTs}^{27}$ as possible. The device consists of a processed copper PCB (milled or etched), cut 3M tape (by hand or laser cutter), and injected with 1-butyl-3-methylimidazolium tetrafluoroborate ([BMIM $]^{+}\left[\mathrm{BF}_{4}\right]^{-}$) Ionic Liquid (IL). This IL is used as a soft sensing medium due to its non-volatility, with benefits such as low vapour pressure, low freezing point, and high flash point. ILs are incompressible allowing them to maintain a constant volume at a constant temperature in a closed volume/chamber sensing device. ${ }^{28}$

By using a low Young's modulus material as the surface of the sensor, an array of electrodes has been positioned to allow for differential position measurements to use in a similar manner to conventional pressure measurements. Due to the incompressible nature of the IL, the shift in weight or pressure can be measured differentially and correlated to a percentage change of the overall pressure measured. It also means the device maintains its volume and does not deflate when used. Such measurements can be used to aid in showing the shift in weight during motion/manipulation of a hand or robot gripper. However, the device is soft and capable of deforming against the object it is in contact with, like that of the human fingertips. As part of the sensor is under pressure, it will expand in the lower pressure regions, increasing the difference between each 
section of the sensor. Thus, the device can indicate in which section of the sensor is under the most pressure. While the device is not under pressure it is possible to use it for temperature measurements in the same way as our previously reported work. $^{29}$

\section{Materials and devices}

IL is used as an electrically conductive sensing medium for our device to facilitate soft sensing capability. In an aqueous ionic solution, the ions carry charge between electrodes. The effective ionic conductance of the IL is expressed by

$$
\sigma_{\mathrm{IL}}=\mu n z F
$$

where $F$ is Faraday constant, $z$ is valence of ion charges, $n$ is concentration of IL, and $\mu$ the mobility of the ions. It can be seen that as the mobility of the ions increases the conductivity will increase.

Using the ionic conductivity from eqn (1), the effective resistance (real component of impedance $Z$ ) between two electrodes in IL can be approximated as

$$
R_{\mathrm{e}}=\frac{s}{l h \sigma_{\mathrm{IL}}},
$$

where $l$ is a constant length of electrode, $s$ is a constant distance between electrodes, and $h$ is the effective height of the IL volume as shown in Fig. 1(b) and (c).

The effects of temperature can be described by the relation between temperature and viscosity, where an increase in temperature will cause a change viscosity. ${ }^{30,31}$ These changes can be related to the change in effective resistance through diffusivity $(D)$, as an increase in diffusivity allows for the ions to propagate quicker, ${ }^{32}$

$$
D=\frac{k_{\mathrm{B}} T}{c \pi \eta},
$$

where $k_{\mathrm{B}}$ - Boltzmann constant, $T$ - absolute temperature, $c$ coefficient of proportionality, and $\eta$ - viscosity.

From eqn (2) and (3), we can show that a change in height $(h)$ under an applied pressure $(P)$ or a change in ion mobility $(\mu)$ due to varying ambient temperature $(T)$,

$$
R_{\mathrm{e}} \propto[h(P) \mu(T)]^{-1},
$$

representing height as a function of pressure, and ion mobility as a function of temperature. From this formula we can see that

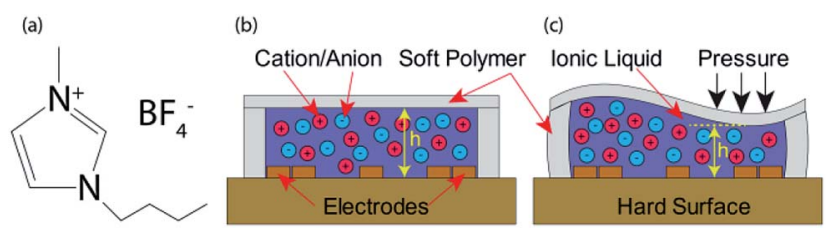

Fig. 1 (a) Chemical structure of $[B M I M]^{+}\left[B F_{4}\right]^{-}$, (b) sensor at rest, (c) sensor under external applied pressure. as the pressure increases, or temperature decreases, the effective resistance will increase.

To select an IL, the physical properties and merits were evaluated on the cost and safety of the IL, where the most expensive ILs were the most inert and safe to handle. A trade-off for a low-cost solution that remained safe to handle if basic PPE and appropriate disposal were used. We purchased $\geq 98 \%$ assay 1-butyl-3-methylimidazolium tetrafluoroborate $\left([\mathrm{BMIM}]^{+}\left[\mathrm{BF}_{4}\right]^{-}\right)$ from Sigma Aldrich. The chemical structures of $[\mathrm{BMIM}]^{+}\left[\mathrm{BF}_{4}\right]^{-}$ is shown in Fig. 1(a). The main benefits and perks in handling centre around the ILs non-volatility and low vapour pressure of $<0.125 \times 10^{-3} \mathrm{hPa}$. A low freezing point of $202 \mathrm{~K}$ and high flash point of $561 \mathrm{~K}$ are well suited for the range of measurements expected in our application ranging from $290 \mathrm{~K}$ to $360 \mathrm{~K}$, well within the safe limits of the material. Electrical conductivity was measured to be $0.95 \mathrm{~S} \mathrm{~m}^{-1}$ which lies between the conductivity range of this $\mathrm{IL}$ reported in literature $\left(0.173 \mathrm{~S} \mathrm{~m}^{-1}\right.$ and $0.35 \mathrm{~S}$ $\mathrm{m}^{-1},{ }^{33}$ and $5.77 \mathrm{~S} \mathrm{~m}^{-1}$ (ref. 34)). This indicates a range of excellent electrical properties for us to propose the design and implementation of a simple sensor structure.

We have shown how the deformation and displacement of a volume of electrically conductive IL covering a set of electrodes is capable of changing the effective resistivity or, in terms of the device, the output impedance/voltage. As the length and distance between electrodes is a design constant, the working principle of the proposed device is based mostly on the deformation of the IL volumes effective height around the electrodes. As the movement of IL is pushed towards one side or another, the impedance between these electrode pairs change dynamically. It can be seen in Fig. 1(c) when pressure is applied to one side of the device, the deformation is reciprocated across the other areas of the device, causing an increase in impedance in the area under pressure and a decrease in those not. The increase in impedance is attributed to the repopulation of charge cation and anion in aqueous solution under pressure.

Physical deformation of the surface area of the sensor under an applied pressure is the main contributing factor in the change of impedance between electrodes (shown in Fig. 1 and 2). By using thick walled $(2.5 \mathrm{~mm})$ soft polymer on the perimeter of the sensor it's possible to maintain a soft/rubbery sensor that is less sensitive to compression and expansion in the sides than the surface area. Due to the low Young's modulus of the soft

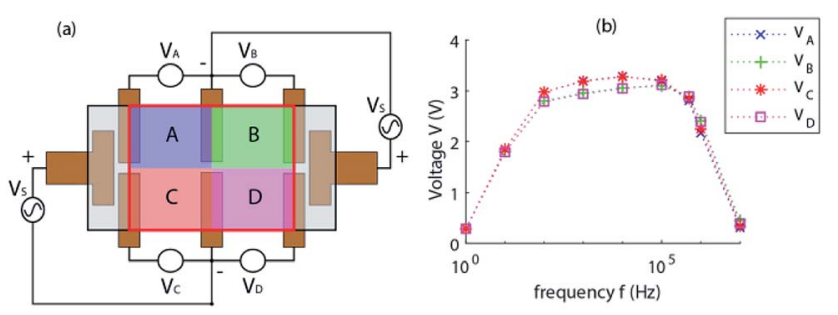

Fig. 2 (a) Sensor design with electrode positioning and proposed measurement circuit configuration. Sensing areas coloured and outlined in red. Grey area is an introduced impedance for voltage measurement and demonstration. (b) Frequency response of adjacent configuration. 
polymer, the surface area of the sensor is very soft and elastic in nature, where compression in one part of the sensor will provide expansion in the other areas. This effect has been used to design a sensor that is capable of showing a difference in pressures between four sensing areas of the one device. By reducing the initial height (depth of the IL layer) the sensitivity of the device will increase. However, an IL layer that is too shallow will result in a shortening range and undesirable linearity, as the membrane will no longer deform after making contact with the electrodes on the bottom layer of the device. An increase in initial height will reduce sensitivity and increase the range. However, a larger IL volume can cause sensor bloating, as it is a sealed device filled with an incompressible liquid.

To perform simple and symmetrical voltage measurements and demonstration using the device, an alternate measurement method was presented (Fig. 2(a)) based on conventional 4-point measurement methods. The device is offset with an internal voltage for direct measurements with an oscilloscope or microcontroller without requiring additional circuitry and configured in a manner to provide symmetrical readings when the device is not under pressure. This offset voltage is introduced when we power the device via $V_{\mathrm{s}}$ (far left and right electrodes in Fig. 2(a)) which is represented by the grey shaded rectangles that are outlined in black. We have performed measurements in this manner to utilise the convenience of the sensor and circuit (as seen in Fig. 6). Due to the very soft and malleable nature of the surface of the sensor, minor asymmetry can be seen between the $V_{\mathrm{A}} \& V_{\mathrm{C}}$ side and the $V_{\mathrm{B}} \& V_{\mathrm{D}}$ side of the device. Frequency of the excitation voltage applied to the sensor shows a high impact on the output magnitude of the device and minor effects on the symmetry. Low frequency measurements show that it is indeed unsuitable to use DC measurements on an ionic solution, with peak symmetry and high response shown at around $500 \mathrm{kHz}$ (Fig. 2(b)).

For testing purposes, a deformable rectangular channel of IL with a height, width, and length of $0.5 \mathrm{~mm} \times 7.0 \mathrm{~mm} \times$ $14.0 \mathrm{~mm}$ respectively, is fabricated on a PCB board. However, it may be possible to adhere the sensor to different surface profiles if fabrication and positioning of the electrodes is not an issue. It should be noted that the surface area for adhesion with the tape used in our work requires a smooth surface to ensure no leakage occurs.

\section{Fabrication}

Copper PCB was fabricated using a router (LPKF ProtoMat S42). The resulting PCB was rough and required gentle sanding (3M 734 P240) before use. Sheets of $0.5 \mathrm{~mm}$ thick tape (3M VHB acrylic foam tape) were measured and cut using a scalpel (Duratool D00298). Connecting wires were soldered before and after cleaning the copper PCB with a citric acid solution and acetone. The first layer, a hollow rectangular cut-out of tape is affixed to the surface of the cleaned PCB, followed by a solid rectangular layer of tape. IL ([BMIM $\left.]^{+}\left[\mathrm{BF}_{4}\right]^{-}\right)$was injected into the cavity by pressing a $0.3 \mathrm{~mm}$ needle (BD Microlance 3) through the second layer into the cavity at an angle to avoid the underside of the second layer from unintentionally sticking to the surface of the PCB. The needle and syringe are repeatedly used to ensure the sensor is filled with the IL solution and all air bubbles are removed. Due to the fine point of the needle used, these holes do not leak the viscous IL substance as long as the sensor isn't put under unnecessary internal or external pressure before the third layer is applied. Upon removal of the needle from the second layer, it was observed that the tape adheres to itself effectively sealing the perforation, this is shown in Fig. 3(f). Finally, holes in the second layer of the sensor are sealed with a third layer of tape. The protective layers on the tape were not removed prior to assembly. This process is further detailed and can be seen in Fig. 3.

\section{Results and discussion}

The fabricated sensor is shown as an inset in Fig. 4. To calibrate the sensor, pressure was applied to the surface area over each sensing area of the device using a $4 \mathrm{~mm} \times 5 \mathrm{~mm}$ piece of acrylic. The force applied to the sensor was measured accurately and simply using a scale (OHAUS Pioneer PA4102) supporting the sensor. An LCR Meter (U1733C) was used to perform precision impedance measurements of the output of each section of the sensor (positions $V_{\mathrm{A}}, V_{\mathrm{B}}, V_{\mathrm{C}}$, and $V_{\mathrm{D}}$ as seen in Fig. 2(a)).

It can be seen from Fig. 4(a) that, when a pressure is applied to section A, the local volume of IL is decreasing causing a large increase in impedance of this section, while the opposing sides $\mathrm{B}$ and $\mathrm{D}$ show slightly decreased impedances. Output from section $\mathrm{C}$ shows a minor increase in impedance as it is close to the area of applied pressure. Similarly, in Fig. 4(b), the most changed signal is $\mathrm{B}$, followed by the opposing sides A and $\mathrm{C}$, where $\mathrm{D}$ is the least effected. This convention can also be seen in Fig. 4(c) and (d). These agree well with the design analysis presented, showing a strong correlation between the change in pressure and output of the device as presented in Fig. 1 and eqn (4), where $R_{\mathrm{e}} \propto[h(P)]^{-1}$. Small differences between measurements can be seen in Fig. 4 due to the position of the applied pressure not being the same between each sensing area. However, the device has clearly shown an excellent ability to distinguish between each pressure sensitive area.

Voltage measurements were performed by exiting the device using a signal generator (Keysight Technologies MSOX3104A)

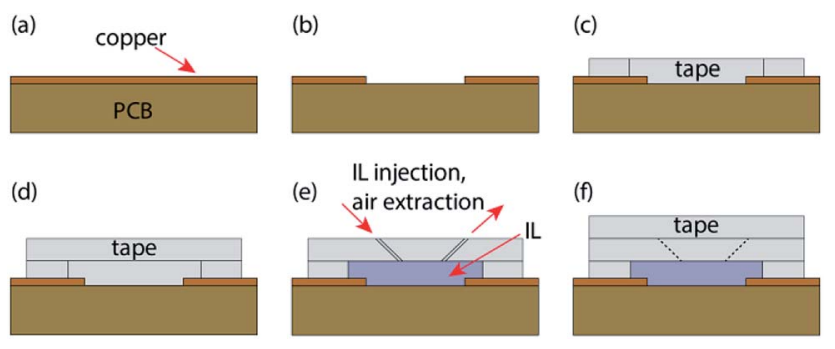

Fig. 3 Cross section of fabrication and assembly process. (a) PCB preparation, (b) copper etching/milling of electrodes, (c) adhesion of $0.5 \mathrm{~mm}$ hollow cut tape, (d) bonding of $0.5 \mathrm{~mm}$ solid cut tape, (e) channel injected with IL, (f) bonding of final $0.5 \mathrm{~mm}$ tape layer. 

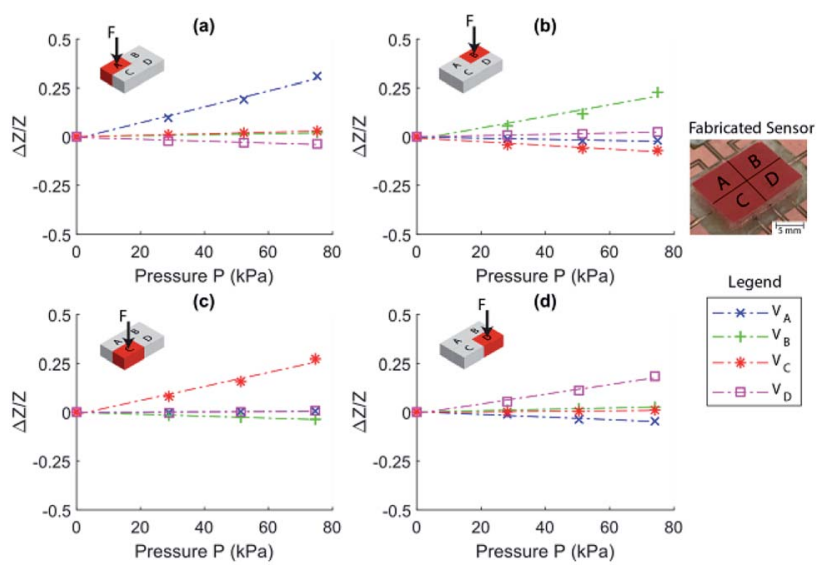

Fig. 4 Relative changes of output impedances when each quadrant of the device is subject to pressure. (a), (b), (c), and (d) Represent the device outputs when quadrant $A, B, C$, or $D$ respectively is under an applied pressure.

supplying $5 \mathrm{Vpp}$ @ $500 \mathrm{kHz}$ and voltage measurements were taken using a four channel oscilloscope (Rigol DG1022). Peakpeak voltages for each section of the device were recorded alongside the applied weight/force data. The relative changes of output voltages $\left(V_{\mathrm{A}}, V_{\mathrm{B}}, V_{\mathrm{C}}\right.$, and $\left.V_{\mathrm{D}}\right)$.

Using this measurement method, we propose a dynamic reference calculated by the total measured value over all four sensing areas $\left(V_{\text {total }}\right)$ relative to each individual value at the same time as measurement. By using a dynamic reference point, it is possible to theoretically diminish the temperature effect on the output of the device. Thus, instead of using a reference voltage from calibration, a ratio is calculated at the time of each measurement. The device consists of a single chamber where, as long as contact with varying temperatures is distributed evenly across the surface of the device, the internal temperature will change at a near-consistent rate through the IL. This change in temperature should evenly and equally affect each of the four sections of the one device. Therefore, at any time, as long as $V_{\text {total }}$ is referenced at the same time at each measurement,

$$
\frac{V_{\mathrm{e}}(1+\alpha \Delta T)}{V_{\text {total }}(1+\alpha \Delta T)},
$$

where $V_{\text {total }}$ is the sum of all four sensing elements $\left(V_{\mathrm{e}}\right)$ at $V_{\mathrm{A}}, V_{\mathrm{B}}$, $V_{\mathrm{C}}$, and $V_{\mathrm{D}}$, will result in a ratio that is independent of temperature effects. This will also make the measurements to be insensitive to the overall change in voltage of the device caused by pressures applied directly perpendicular to the surface of the whole sensing area.

The desired output of the device is mathematically shown to be temperature independent. However, the presented device uses an IL that has reported high temperature/viscosity relation $^{30,32}$ and thus a high Temperature Coefficient of Impedance $(\mathrm{TCZ}){ }^{33}$ In our previous work we have shown that [BMIM $]^{+}\left[\mathrm{BF}_{4}\right]^{-}$IL is capable of a very high TCZ of up to $-17300 \mathrm{ppm} \mathrm{K}{ }^{-1} \cdot{ }^{29}$ The main contributing factor to these measurements was determined to be the ILs diffusivity in relation to viscosity and temperature. Similarly, when our current device is under a known pressure/load (e.g. no load), it is possible to use one (or all) cells of the device to make ambient temperature measurements. Once the system is under load, these measurements become unreliable due to the acting pressure and temperature on the device. We hypothesise that the change in viscosity may also have an impact on the sensitivity of the device to pressure due to the slight change in the materials deformability.

The effect of pressure $\mathrm{d} P_{\mathrm{e}}$ at a sensing element cause the locally average deformation $\mathrm{d} \varepsilon_{\mathrm{e}}$, which changes the voltage of the element to $V_{\mathrm{e}}\left(1+\beta_{\mathrm{e}} \mathrm{d} \varepsilon_{\mathrm{e}}\right)$ (where $\beta_{\mathrm{e}}$ is the coefficient representing the linear relation between mechanical deformation and the output voltage, as shown in Fig. 4), will also affect the voltage throughout the device sensed by other elements $i_{\mathrm{th}}, V_{\mathrm{e}_{i}}(1$ $+\beta_{i} \mathrm{~d} \varepsilon_{i}$ ). By setting the initial voltage equally at each element (see sensor symmetricity shown in Fig. 2(a)), the sum of voltage is simplified as

$$
V_{\text {total }_{\mathrm{p}}}=V_{\text {total }_{0}}+V_{i} \sum \beta_{i} \mathrm{~d} \varepsilon_{i},
$$

where $V_{i} \sum \beta_{i} \mathrm{~d} \varepsilon_{i}$ is the total change of voltage across the whole deformed area of the sensor surface. As the compression of the wall is negligible in comparison with the deformation of the wall itself, and because the ionic liquid is incompressible in the range of test pressure, $V_{i} \sum \beta_{i} \mathrm{~d} \varepsilon_{i}$ cancels out and the total voltage would remain a constant. This is shown experimental data in Fig. 5 where the total voltage change is only $3-4 \%$. Therefore, by measuring $V_{\mathrm{e}} / V_{\text {total }}$, the effect of temperature is filtered, and the effect of local deformation under pressure is sorted out. It should be noted that in practice the sensor does not have rigid sides, and there is a non-sensitive area on either side of the sensing zone, these have an effect on the output of the system and explain why the sum of output voltages does not remain perfectly constant. In a perfect system with rigid walls, it could

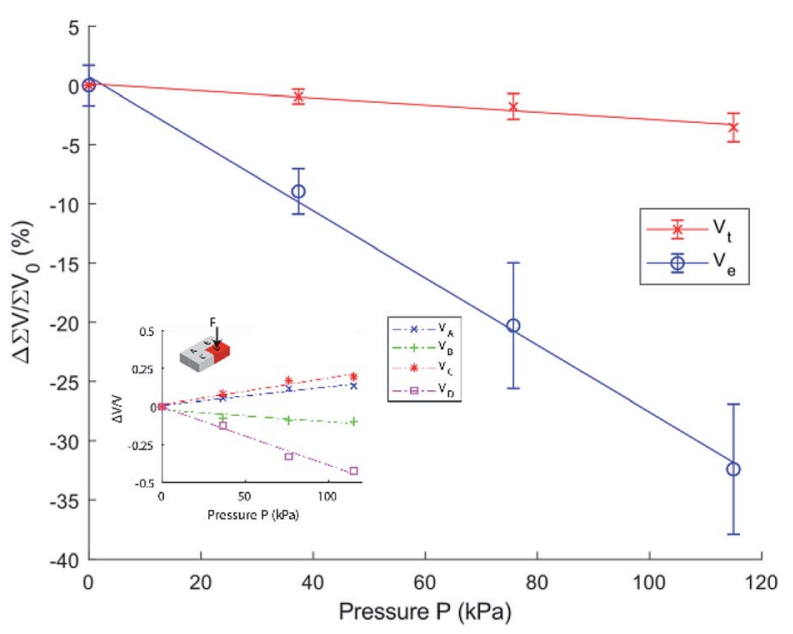

Fig. 5 Change in sum of voltages $\left(V_{t}\right)$ as pressure changes, averaged by four measurements with pressure applied at each sensing element $\left(V_{\mathrm{e}}\right)$. Error bars represent std over 10 measurements. Inset shows sample of voltage measurement of the device for one element $\left(V_{d}\right)$. 

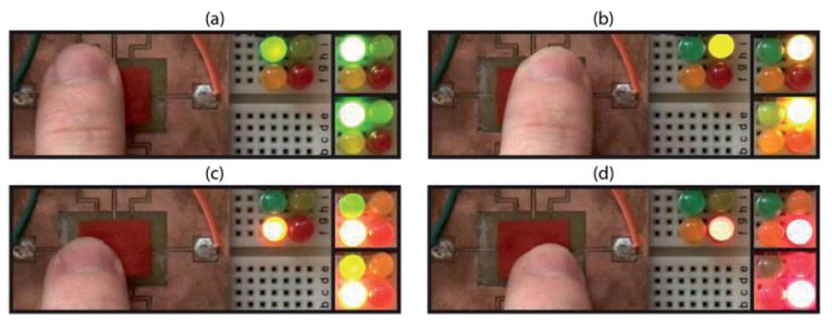

Fig. 6 Touch applied to sensing areas A (green), B (yellow), C (orange), and $D$ (red) with LED indication of total applied pressure at each point Low, medium, and high pressures demonstrated for each sensing element via brightness of LED.

be observed that the change in the sum of voltages measured in the system is constant.

Fig. 5 shows that the system is capable of a sensitivity of $-0.28 \% \mathrm{kPa}^{-1}$ which is comparable to $0.039 \mathrm{kPa}^{-1}, 350.059$ $\mathrm{kPa}^{-1},{ }^{36}$ and $0.023-0.068 \mathrm{kPa}^{-1}$ (ref. 37) and even shows higher sensitivity than $0.23 \times 10^{-3} \mathrm{kPa}^{-1}$ (ref. 38) and that of conventional MEMS pressure sensors (e.g. $0.2 \times 10^{-6} \mathrm{kPa}^{-1}$ to $0.035 \times 10^{-3} \mathrm{kPa}^{-1}$ (ref. 39)) whose fabrication processes are a multitude more complex than our presented work. It can be seen that the voltage is decreasing rapidly instead of increasing with increased pressure, this can be attributed to the introduced impedances shown in Fig. 2.

Furthermore, by using this measurement method, a microcontroller with a synchronous sampling frequency can be used to make all the measurements and supply the constant AC supply (square wave) to the sensor with no additional circuitry. An example of this is shown in Fig. 6, using an Arduino Leonardo and simple breadboard circuit with four LEDs, to demonstrate the sensors capabilities.

With this elementary setup we were capable of visually representing pressure distribution using four LEDs. It can be seen in Fig. 6(c) that the green LED is active while applying pressure to sensing element $\mathrm{C}$ (orange), it's apparent by the finger position that the pressure is not being applied in the exact same location as seen in Fig. 6(d) by comparison. Thus, it is expected that section A shows some response to the applied pressure in section $\mathrm{C}$.

\section{Conclusions}

We successfully demonstrated a soft IL-based pressure sensor which is capable of highly sensitive detection of touching points. The chosen IL $\left([\mathrm{BMIM}]^{+}\left[\mathrm{BF}_{4}\right]^{-}\right)$showed excellent stability and electrical properties for soft tactile and pressure sensing applications. Based on our original measurement circuit configuration and signal processing method, the output symmetry is unaffected by frequency, whereas conventional 4point measurements in this configuration would be asymmetric. The device is shown to be insensitive to temperature and perpendicular pressure applied to the whole sensing surface, with a low impact on the overall output of the system, but localised pressure creates large output changes. Thanks to the smart structure design and the conservative volume nature of
IL, the touching point can be detected with high sensitivity. These results indicate the potential of using $[\mathrm{BMIM}]^{+}\left[\mathrm{BF}_{4}\right]^{-}$for soft sensing devices with high performance of multi-point touch functions.

\section{Conflicts of interest}

There are no conflicts to declare.

\section{Acknowledgements}

This work was performed in part at the Queensland node of the Australian National Fabrication Facility, a company established under the National Collaborative Research Infrastructure Strategy to provide nano and micro-fabrication facilities for Australia's researchers.

\section{Notes and references}

1 M. Wehner, R. L. Truby, D. J. Fitzgerald, B. Mosadegh, G. M. Whitesides, J. A. Lewis and R. J. Wood, Nature, 2016, 536, 451-455.

2 D. Rus and M. T. Tolley, Nature, 2015, 521, 467-475.

3 J. Morrow, H.-S. Shin, C. Phillips-Grafflin, S.-H. Jang, J. Torrey, R. Larkins, S. Dang, Y.-L. Park and D. Berenson, Improving Soft Pneumatic Actuator fingers through integration of soft sensors, position and force control, and rigid fingernails, 2016 IEEE International Conference on Robotics and Automation (ICRA), Stockholm, 2016, pp. 50245031, DOI: 10.1109/ICRA.2016.7487707.

4 A. M. Almassri, W. Z. W. Hasan, S. A. Ahmad, A. J. Ishak, A. M. Ghazali, D. N. Talib and C. Wada, J. Sens., 2015, 2015, 846487.

5 M. Zhu, Z. Wang, S. Hirai and S. Kawamura, Design and fabrication of a soft-bodied gripper with integrated curvature sensors, 2017 24th International Conference on Mechatronics and Machine Vision in Practice (M2VIP), Auckland, 2017, pp. 1-6, DOI: 10.1109/M2VIP.2017.8211521.

6 T. Dinh, H. P. Phan, T. K. Nguyen, A. Qamar, A. R. M. Foisal, T. N. Viet, C. D. Tran, Y. Zhu, N. T. Nguyen and D. Viet Dao, J. Mater. Chem. C, 2016, 4, 10061-10068.

7 G. Ge, Y. Cai, Q. Dong, Y. Zhang, J. Shao, W. Huang and X. Dong, Nanoscale, 2018, 10, 10033-10040.

8 T. Yang, D. Xie, Z. Li and H. Zhu, Mater. Sci. Eng., R, 2017, 115, 1-37.

9 R. K. Kramer, C. Majidi and R. J. Wood, Wearable tactile keypad with stretchable artificial skin, 2011 IEEE International Conference on Robotics and Automation, Shanghai, 2011, pp. 1103-1107, DOI: 10.1109/ICRA.2011.5980082.

10 B. Nie, R. Li, J. D. Brandt and T. Pan, Lab Chip, 2014, 14, 1107-1116.

11 J. C. Yeo, J. Yu, K. P. Loh, Z. Wang and C. T. Lim, ACS Sens., 2016, 1, 543-551.

12 C. Larson, B. Peele, S. Li, S. Robinson, M. Totaro, L. Beccai, B. Mazzolai and R. Shepherd, Science, 2016, 351, 1071-1074.

13 T. Dinh, H. P. Phan, A. Qamar, N. T. Nguyen and D. V. Dao, $R S C$ Adv., 2016, 6, 77267-77274. 
14 R. Bogue, Industrial Robot: An International Journal, 2016, 43, 258-263.

15 V. A. Ho, D. V. Dao, S. Sugiyama and S. Hirai, IEEE Trans. Rob., 2011, 27, 411-424.

16 R. M. Pierce, E. A. Fedalei and K. J. Kuchenbecker, A wearable device for controlling a robot gripper with fingertip contact, pressure, vibrotactile, and grip force feedback, 2014 IEEE Haptics Symposium (HAPTICS), Houston, TX, 2014, pp. 19-25, DOI: 10.1109/HAPTICS.2014.6775428.

17 S. Levine, P. Pastor, A. Krizhevsky, J. Ibarz and D. Quillen, Int. J. Robotics Res., 2018, 37, 421-436.

18 T. B. Jørgensen, S. H. N. Jensen, H. Aanæs, N. W. Hansen and N. Krüger, J. Intell. Robotic Syst., 2019, 94, 81.

19 M. H. Lee and H. R. Nicholls, Mechatronics, 1999, 9, 1-31.

20 M. R. Cutkosky and W. Provancher, in Springer Handbook of Robotics, ed. B. Siciliano and O. Khatib, Springer International Publishing, Cham, 2016, pp. 717-736, DOI: 10.1007/978-3-319-32552-1_28.

21 A. Ahmed, S. L. Zhang, I. Hassan, Z. Saadatnia, Y. Zi, J. Zu and Z. L. Wang, Extreme Mechanics Letters, 2017, 13, 25-35.

22 Y. Yang, M. Y. Cheng, W. Y. Chang, L. C. Tsao, S. A. Yang, W. P. Shih, E. Y. Chang, S. H. Chang and K. C. Fan, Sens. Actuators, 2008, 143, 143-153.

23 J. Li, R. R. Bao, J. Tao, Y. Y. Peng and C. F. Pan, J. Mater. Chem. C, 2018, 6, 11878-11892.

24 J. Engel, J. Chen and C. Liu, J. Micromech. Microeng., 2003, 13, 359-366.

25 J.-B. Chossat, H.-S. Shin, Y.-L. Park and V. Duchaine, Journal of Mechanisms and Robotics, 2015, 7, 021008.

26 T. Someya, Y. Kato, T. Sekitani, S. Iba, Y. Noguchi, Y. Murase, H. Kawaguchi and T. Sakurai, Proc. Natl. Acad. Sci. U. S. A., 2005, 102, 12321-12325.
27 V. T. Dau, T. Yamada, D. V. Dao, B. T. Tung, K. Hata and S. Sugiyama, Microelectron. J., 2010, 41, 860-864.

28 K. R. Harris, M. Kanakubo and L. A. Woolf, J. Chem. Eng. Data, 2007, 52, 2425-2430.

29 J. Fastier-Wooller, R. Yoshikawa, T. Dinh, V. Dau, H.-P. Phan, A. Teo, S. H. Tan and D. V. Dao, in Sustainable Design and Manufacturing 2018, 2019, ch. 19, pp. 184-192, DOI: 10.1007/978-3-030-04290-5_19.

30 R. Brookes, A. Davies, G. Ketwaroo and P. A. Madden, J. Phys. Chem. B, 2005, 109, 6485-6490.

31 K. R. Harris, M. Kanakubo and L. A. Woolf, J. Chem. Eng. Data, 2007, 52, 2425-2430.

32 C. Schreiner, S. Zugmann, R. Hartl and H. J. Gores, J. Chem. Eng. Data, 2009, 55, 1784-1788.

33 M. Galiński, A. Lewandowski and I. Stępniak, Electrochim. Acta, 2006, 51, 5567-5580.

34 W. W. Liu, T. Y. Zhao, Y. M. Zhang, H. P. Wang and M. F. Yu, J. Solution Chem., 2006, 35, 1337-1346.

35 S. Chun, Y. Kim, H. S. Oh, G. Bae and W. Park, Nanoscale, 2015, 7, 11652-11659.

36 Y. H. Wu, H. Z. Liu, S. Chen, X. C. Dong, P. P. Wang, S. Q. Liu, Y. Lin, Y. Wei and L. Liu, ACS Appl. Mater. Interfaces, 2017, 9, 20098-20105.

$37 \mathrm{X} . \mathrm{Wu}, \mathrm{Y}$. Han, X. Zhang, Z. Zhou and C. Lu, Adv. Funct. Mater., 2016, 26, 6246-6256.

38 D. J. Lipomi, M. Vosgueritchian, B. C. Tee, S. L. Hellstrom, J. A. Lee, C. H. Fox and Z. Bao, Nat. Nanotechnol., 2011, 6, 788-792.

39 H. P. Phan, D. V. Dao, K. Nakamura, S. Dimitrijev and N. T. Nguyen, J. Microelectromech. Syst., 2015, 24, 1663-1677. 